\title{
EDITORIAL
}

\section{Is Early Infarct Artery Patency the Goal or Not?}

\author{
ERIC R. BATES, M.D. \\ From the Division of Cardiology, University of Michigan Medical Center, Ann Arbor, Michigan
}

\section{Introduction}

Whereas the majority of influential cardiologists initially embraced the biotechnology achievement and TIMI I superiority ${ }^{1}$ associated with tissue plasminogen activator (tPA), now the previously beleaguered streptokinase and APSAC advocates are gaining their measure of revenge after years of being intellectually suppressed by the tPA advocates. ${ }^{2-4}$ Early infarct artery patency at whatever cost (or expense) because "time is muscle" is no longer supposed to be the driving hypothesis behind selecting a lytic agent because comparative trials have not demonstrated drug related differences in left ventricular ejection fraction or mortality. ${ }^{5}$ Rather, sustained patency at 24 hours is being touted as more predictive of clinical outcome even as a major effort continues to investigate prehospital administration of thrombolytic therapy in order to save precious minutes initiating therapy. ${ }^{6}$ As in any debate, both sides are selectively quoting data to support their respective arguments. So what patency rates are we really comparing and is early infarct artery patency the goal or not?

A number of differences in study design influence the determination of patency rates in reperfusion reports. First, studies using clinical markers of reperfusion including relief of chest pain,

Address for reprints: Eric R. Bates, M.D., B1F245 University Hospital, 1500 East Medical Center Drive, Ann Arbor, MI 48109-0022. Fax: (313) 936-7641.

Submitted for publication January 15, 1992; accepted for publication January 15, 1992. normalization of ST segment elevation, or bursts of accelerated idioventricular rhythm have occasionally been included with angiographic studies, even though they do not accurately reflect patency. ${ }^{7}$ Coronary angiography should be the only standard by which to evaluate reperfusion. Second, doses and infusion times of some thrombolytic agents have varied widely. Higher doses or more rapid infusion times have improved patency rates in studies with alteplase and longer infusions may have reduced reocclusion rates in other studies. ${ }^{8}$ Third, time to treatment is important because organization of the thrombus begins to occur after the first few hours, decreasing acute patency rates in patients who present later for treatment. ${ }^{1}$ This appears more important when streptokinase or APSAC are used. Fourth, time to angiography influences results. Ninety-minute patency rates are "snapshots" in time, arbitrarily chosen because of the logistics involved in transporting the patient to the cardiac catheterization laboratory. Later evaluation will result in higher patency rates because of continued thrombolysis. Fifth, the hydrostatic force of injecting contrast in the coronary artery can result in occasional patency. The TAMI trials use the fourth angiographic injection as the endpoint rather than the first injection, increasing patency rates by approximately $5 \%$. Finally, studies with fewer than 50 patients should not be given equal weight to studies with $100-200$ patients.

A recent review ${ }^{8}$ only analyzed studies using standard dosing (alteplase, $100 \mathrm{mg} / 3 \mathrm{hrs}$; APSAC, 30 units $/ 5 \mathrm{~min}$; duteplase, $0.6 \mathrm{MU} / 4 \mathrm{hrs}$; saruplase, $80 \mathrm{mg} / 1 \mathrm{hr}$; streptokinase, 1.5 million units/ 
$1 \mathrm{hr}$ ), angiography, 90-minute patency, and mean symptom duration 2.5 to 4 hours. Whereas streptokinase had the lowest 90 -minute patency rate (53\%); alteplase, anistreplase, duteplase, and saruplase were equivalent $(68 \%, 72 \%, 69 \%$, and $72 \%$, respectively). Note that the alteplase patency rate with conventional dosing is lower than generally appreciated and no different from the duteplase result, as emphasized by the ISIS-3 investigators. Previous reviews quoting higher patency rates for alteplase were biased by including studies that used weight adjusted dosing schedules where more than $1 \mathrm{mg} / \mathrm{kg}$ of alteplase was given by 90 minutes. On the other hand, only 70 $\mathrm{mg}$ of the $100 \mathrm{mg}$ conventional dose is infused by the time of the 90 -minute angiogram. When the full thrombolytic dose is given before the 90 -minute angiogram (front-loaded alteplase), as it is with the other thrombolytic agents, an $85 \%$ patency rate is obtained, the highest rate yet reported for thrombolytic monotherapy. Patency rates are higher with combination thrombolytic therapy (alteplase/streptokinase, alteplase/urokinase) than conventional dose monotherapy, but not as high as with front-loaded tPA therapy. By 24 hours, equivalent patency rates of $85 \%-90 \%$ have been documented for all agents.

Sherry and $\mathrm{Marder}^{2}$ have argued that all thrombolytic agents are equally effective. In the early hours of myocardial infarction, when myocardium at risk can potentially be salvaged, they suggest that the agents can dissolve fresh fibrin equally well and achieve equivalent patency rates. As fibrin ages, it becomes more resistant to dissolution. Higher acute patency will be achieved with IPA relative to streptokinase because of fibrin specificity, but significant salvage is no longer possible within this time frame. Therefore, equivalent results between agents with respect to left ventricular ejection fraction and mortality would again be expected from the open artery hypothesis because sustained patency rates are equivalent several hours after treatment.

However, it has yet to be proven that patency rates are equivalent among the agents in the first few hours of myocardial infarction. Moreover. when studies with similar methodologies are evaluated, equivalent patency rates have not been achieved 3 hours after initiating therapy, as sug- gested by Sherry and Marder $^{2}$; tPA rates are still 5-15 percentage points higher. ${ }^{8}$

Spontaneous patency develops in one third of patients during the first day of myocardial infarction when thrombolytic agents are not used and two thirds of infarct arteries ultimately become patent. The acute benefit has not been carefully evaluated, but later prognostic advantage has been claimed for spontaneous patency. ${ }^{9,10}$

Before final patency status is determined, thrombus dissolution and reformation can occur several times. Higher fibrinogen levels may contribute to rethrombosis; lower fibrinogen levels and the resulting increase in the products of fibrinogen degradation that exert potent antiplatelet and anticoagulant effects may favor continued patency. In support of this concept is the suggestion that patients treated with tPA have a higher incidence of recurrent temporary thrombosis as measured by the continuous 12-lead ST segment monitor, compared with streptokinase. ${ }^{11}$ If further cellular damage is caused by recurrent occlusion, this could explain why no difference in left ventricular function or mortality has been shown to date in clinical trials despite differences in early patency rates between the agents.

Therapeutic anticoagulation appears to be critically important in maintaining patency achieved with alteplase. Patency rates $>90 \%$ were achieved angiographically when the activated partial thromboplastin time (aPTT) was consistently maintained over 2.0 times baseline in the ECSG- $6^{12}$ and HART ${ }^{13}$ trials. Rates were more than 20 points lower when at least one aPTT measurement was $<60$ seconds. In contrast, no significant patency advantage was found when intravenous heparin was added to APSAC therapy. ${ }^{14}$

Angiographic reocclusion of the infarct artery doubles hospital mortality. ${ }^{15}$ Half of these events occur in the first 24 hours and half of the 7-day reocclusion events are silent. In the absence of intravenous heparin adjunctive therapy, reocclusion rates of $20 \%-30 \%$ occur following alteplase therapy. ${ }^{16.17}$ Reocclusion rates associated with subcutaneous heparin adjunctive therapy are not known. Seven-day reocclusion rates of 10\%-15\% occur when aspirin and intravenous heparin are given with alteplase and fall below $10 \%$ with combination thrombolytic therapy and intravenous heparin. ${ }^{8}$ Seven-day reocclusion rates for strep- 


\section{EARLY INFARCT ARTERY PATENCY}

tokinase and APSAC have not been well defined. The impact of more powerful antiplatelet therapy (monoclonal antibodies) and anticoagulant therapy (hirudin) on reocclusion rates are being studied by the TAMI and TIMI groups.

Reperfusion momentum overcomes the tendency for reocclusion in the first 24 hours because 24-hour patency rates are higher than 90minute patency rates. However, patency rates decrease by approximately $10 \%$ from 24 hours to 7 days. ${ }^{8}$ Interestingly, it appears that late reocclusion occurs in another $20 \%$ of patients by 3 months. ${ }^{18}$ These events may not carry the same clinical risk as early reocclusion. Perhaps collateral circulation formation makes many of these events clinically silent or they are associated with completed infarcts.

It seems premature to conclude that all thrombolytic drugs are equivalent and that further mechanistic evaluations are redundant. ${ }^{3,19}$ Rather, it may be that we are finally knowledgable enough to ask the most important questions and design the appropriate studies to answer them. The GUSTO trial, for instance, will attempt to determine whether earlier or more sustained infarct artery patency can improve clinical outcome compared with intravenous streptokinase and subcutaneous heparin by studying intravenous streptokinase and intravenous heparin; weight adjusted, front-loaded tPA, and intravenous heparin; and the combination of TPA and streptokinase plus intravenous heparin. Ninetyminute, 180-minute, and 24-hour patency rates will be determined to accurately define acute patency rates in a randomized trial format with currently available therapy and correlate them with clinical outcome. Moreover, by defining 7-day patency rates, reocclusion rates can be fairly compared.

Besides investigating strategies to open the infarct artery earlier or keep it open for 7 days, it will be important to see whether interventions that potentially reduce the number or duration of intermittent reocclusions ("cyclic flow variations" in the animal literature ${ }^{20}$ ) in the early hours after thrombolysis, such as intravenous heparin (GUSTO), combination thrombolytic therapy (GUSTO, TIMI-4), antiplatelet monoclonal antibodies (TAMI-8), or hirudin (TIMI-5, TIMI-6) improve left ventricular function. If there is a positive impact, we may ultimately be able to validate our initial paradigm, which suggested that acute patency preserves left ventricular function and reduces morbidity and mortality. Then we could justify scientifically and economically the intense efforts currently in progress to improve antiplatelet, anticoagulant, and thrombolytic strategies and to decrease time to treatment in patients with acute myocardial infarction.

\section{References}

1. The TIMI Study Group. The Thrombolysis in Myocardial Infarction (TIMI) trial: Phase I findings. N Engl J Med 1985; 312:923-926.

2. Sherry S, Marder VJ. Streptokinase and recombinant tissue plasminogen activator (rt-PA) are equally effective in treating acute myocardial infarction. Ann Intern Med 1991; 114:417-423.

3. Vogel JHK. Thrombolytic therapy of myocardial infarction-it's time for science, not marketing: The perspective of a practicing cardiologist. Clin Cardiol 1991; 14:548.

4. Anderson JL. Overview of patency as an endpoint of thrombolytic therapy. Am J Cardiol 1991; 67:11E-16E.

5. Yusuf S, Sleight P, Held P, et al. Routine medical management of acute myocardial infarction. Lessons from overviews of recent randomized controlled trials. Circulation 1990; 82(Suppl. II):II-117-II-134.

6. Kouralenko T, Kereiakes DJ, Gibler WB. Prehospital diagnosis and treatment of acute myocardial infarction: A critical review. Am Heart J 1992; 123:181-190.

7. Califf RM, O'Neill WW, Stack RS, et al. Failure of simple clinical measurements to predict perfusion status after intravenous thrombolysis. Ann Intern Med 1988; 108:658-662.

8. Bates ER. Is survival in acute myocardial infarction related to thrombolytic efficacy or the open artery hypothesis? A controversy to be investigated with GUSTO. Chest (In press).

9. Cigarroa RG, Lange RA, Hillis LD. Prognosis after acute myocardial infarction in patients with and without residual antegrade coronary blood flow. Am J Cardiol $1989 ; 64: 155-160$.

10. Lange RA, Cigarroa RG, Hillis LD. Influence of residual antegrade coronary blood flow on survival after myocardial infarction in patients with multivessel coronary artery disease. Cor Artery Dis 1990; 1:56-63.

11. Kwon K, Freedman SB, Wilcox I, et al. The unstable ST segment early after thrombolysis for acute infarction and its usefulness as a marker of recurrent coronary occlusion. Am J Cardiol 1991; 67:109-115.

12. Simoons ML. Patency, recurrent ischemia, reocclusion, heparin, and repeated therapy with alteplase: The ECSG heparin trial. Presented at the George Washington University Thombolysis and Interventional Therapy in Acute Myocardial Infarction Symposium, Anaheim, CA, November 10, 1991.

13. Ross AM. Relationship of anticoagulation to coronary 


\section{BATES}

patency after rt-PA therapy: Further observations from HART. Presented at the George Washington University Thombolysis and Interventional Therapy in Acute Myocardial Infarction Symposium. Anaheim, CA, November 10, 1991.

14. O'Connor CM, Meese R, Navetta F, et al. A randomized trial of heparin in conjunction with anistreplase (APSAC) in acute myocardial infarction. (abstract) Circulation 1991; 84(Suppl. II):II-571.

15. Ohman EM, Califf RM. Topol EJ, et al. Consequences of reocclusion after successful reperfusion therapy in acute myocardial infarction. Circulation 1990; 82:781-791.

16. Hsia J, Hamilton WP. Kleiman N, et al. A comparison between heparin and low-dose aspirin as adjunctive therapy with tissue plasminogen activator for acute myocardial infarction. N Engl J Med 1990; 323: 1433-1437.

17. Bleich SD, Nichols TC, Schumacher RR, et al. Effect of heparin on coronary arterial patency after thrombolysis with tissue plasminogen activator in acute myocardial infarction. Am J Cardiol 1990; 66:1412-1417.

18. Meijer A, Werter CJ, Verhergst FWA. The APRICOT study: Coumarin versus aspirin in the prevention of reocclusion after successful thrombolysis, a prospective placebo-controlled angiographic study. (abstract) Circulation 1991: 84(Suppl. II):II-571.

19. Grines CL, DeMaria AN. The GUSTO trial: Is it necessary? J Interven Cardiol 1991; 4:149-153.

20. Folts JD, Gallagher K, Rowe GG. Blood flow reductions in stenosed canine coronary arteries: Vasospasm or platelet aggregation? Circulation 1982; 65:248-255. 